\title{
HemPHL: A Personal Health Library and mHealth Recommender to Promote Self- Management of Hemophilia
}

\author{
Olufunto A. OLUSANYA ${ }^{\mathrm{a}, 1}$, Nariman AMMAR ${ }^{\mathrm{a}}$, Whitney S. BRAKEFIELD ${ }^{\mathrm{a}, \mathrm{b}}$, Mabel \\ CRESCIONI $^{\mathrm{c}}$, Janet CHUPKA ${ }^{\mathrm{c}}$, DeBran TARVER ${ }^{\mathrm{c}}$ and Arash SHABAN-NEJAD ${ }^{\mathrm{a}}$ \\ ${ }^{a}$ University of Tennessee Health Science Center-Oak Ridge National Laboratory \\ Center for Biomedical Informatics, Department of Pediatrics, Memphis TN, USA \\ ${ }^{\mathrm{b}}$ The Bredesen Center for Data Science, University of Tennessee, Knoxville. TN, USA \\ ${ }^{c}$ Hemophilia Federation of America, Washington DC, USA
}

\begin{abstract}
Hemophilia is a rare inherited bleeding disorder characterized by the blood's inability to clot and could result in potentially life-threatening spontaneous bleeding into joints, organs, and tissues. Moreover, long-term management of this chronic disease is complex and costly. Current scientific evidence demonstrates that personalized digital health technologies could promote and facilitate the selfmanagement of chronic diseases. This study introduces HemPHL a Personal Health Library and mHealth Recommender platform to gather, manage, and exchange tailored health information and recommendations to facilitate self-management and home therapy among individuals with hemophilia. The proposed digital health solution will adopt novel data science, artificial intelligence tools and techniques to manage and use information, as well as promote best practices for health education to enable patients to make informed decisions about their health. To accomplish this, an array of complex health and non-health information will be obtained from multidimensional sources to develop a secure, single access point of information for patient use. Patient's access to personalized health information could harness their engagement and independence as well as empower them to remotely monitor their health progress and improve compliance with treatment plans. This hemophiliafocused, user-centered app can markedly improve patients' clinical outcomes and overall quality of life.
\end{abstract}

Keywords. Personal Health Library, Mobile Health, Digital Health, Hemophilia, Disease Self-management, Quality of life.

\section{Introduction}

Hemophilia is a rare inherited X-linked bleeding disorder characterized by the blood's inability to clot and recurrent spontaneous hemorrhagic events. Hemophilia could result in potentially life-threatening complications such as spontaneous bleeding into joints, soft tissues (e.g., joint arthropathy), and the central nervous system (e.g. intracerebral hemorrhage) [1]. Before the early 1990s, blood transfusion-transmitted infectious diseases such as human immunodeficiency virus (HIV), hepatitis B, and C represented a significant cause of hemophilia-related morbidity and mortality in the United States [2].

\footnotetext{
${ }^{1}$ Corresponding Author, Olufunto A. Olusanya, Centre for Biomedical Informatics, 50 N. Dunlap Street, Memphis, TN 38103; E-mail: oolusan1@uthsc.edu.
} 
Additionally, adverse reactions to treatment therapies, disabling chronic pain, impaired quality of life, and premature deaths have been linked to this genetic disease [1].

Over the last decade, therapeutic advances in treatment strategies and multidisciplinary care have revolutionized the management of hemophilia; reduced comorbidities and hospitalization rates; and transformed perceptions of this once-deadly illness into a chronic, manageable condition. Accordingly, these advanced measures to improve standard and access to care have significantly prevented the incidences of blood product-related comorbidities and therefore should translate to improved patients' clinical outcomes, fewer emergency room visits, and better quality of life. However, despite this therapeutic progress, long-term management of hemophilia is intricate and associated with significant cost implications, and lifelong adverse impacts on patients and caregivers [3]. The substantial burden on each patient in the form of direct costs i.e. hospitalizations, drug therapies, etc., and indirect costs i.e. absenteeism could average over $\$ 140,000$ per year [4]. Self-management supports the effective treatment of lifelong medical conditions and encompasses, "individuals taking on tasks to deal with medical management, role management, or emotional aspects of their condition" [5]. Patients are increasingly taking ownership of their health, personal data and seeking information from diverse sources e.g. Online social media. Since the standard of care for hemophilia is characterized by infusions of prophylactic clotting therapies in a non-clinical setting e.g., patient's home [6], a comprehensive, novel, personalized digital health platform could enable patients to access and exchange their health information, and remotely monitor their bleeding disorders [7]. Accordingly, this study reports a work-inprogress on the design and development of HemPHL, a mobile health application (mHealth app) that utilizes a Personal Health Library (PHL) previously introduced [810], to gather, manage and deliver tailored recommendations to facilitate selfmanagement and treatment adherence among populations diagnosed with hemophilia.

\section{Methods}

\subsection{The Personal Health Library (PHL)}

The PHL is a consumer digital health platform that facilitates the retrieval, collection, organization, utilization, storage and exchange of patient's health information. The PHL can be used by different digital health tools to improve health decision-making and selfcare management. Specifically, the PHL enables patients to construct, and manage a wide range of their health-related information. The HemPHL app will integrate dynamic information, collected from patients' activities, choices, and preferences with relevant contextual knowledge to generate personalized recommendations [11]. These evidencebased recommendations, empower patients through health education, promote best practices, and enable patients to make informed timely decisions about their health.

Sources of Data Knowledge

HemPHL collects and integrates data from an array of complex health and non-health information, in different formats and through different modalities. In addition to clinical data stored in patients' electronic health records (EHRs), HemPHL collects multidimensional social determinants of health $(\mathrm{SDoH})$ data from population-level neighborhood characteristics, as well as Observations of Daily Living (ODL) data from physical activity trackers, electronic subscription and browsing behavior, etc. 
Technological Infrastructures: HemPHL transforms the collected data and information into a machine-readable format using Semantic Web technologies that facilitate interoperability between the PHL and other platforms as well as incorporate patient's personal health knowledge graphs (PHKGs) and global Web-scale knowledge graphs. By implementing distributed privacy and security mechanisms, HemPHL will encourage patients to share their data. The more data is shared, the more knowledge and insights are obtained (directly or inferred) to support self-management practices. HemPHL also utilizes Linked Data principles, which enable dynamic knowledge discovery.

Enabling Hybrid Recommendations: HemPHL can utilize the integrated data and knowledge to provide hybrid (content and context-based) recommendations. To capture context, the app uses ontologies and semantics provided through the PHL. Also, the collected ODL and SDoH data provide explanations and justifications for the recommendations by providing evidence (e.g., provenance links) from trusted sources (e.g., scientific literature, public health agency websites, etc.).

\subsection{Use Case Scenarios}

The features provided by the proposed digital health solution are portrayed in a series of use case scenarios that involve three users, including two patients and one caregiver.

- Scenario 1: A caregiver uses the HemPHL app to obtain information on health resources and educational materials, and for video conferencing as she remotely monitors her son's bleeding episodes and prophylactic factor infusions to prevent further bleeding episodes. She also uses the app for triage and follow-up of her son's clinical condition thereby, potentially reducing an emergency room visit.

- Scenario 2: A patient uses the social features of his app to join support groups and communities to acquire relevant health information, share coping strategies on maintaining a mental health balance, and physical function as well as, facilitate adherence to home therapies/medications, ultimately, improving quality of life.

- Scenario 3: Another patient uses the app to facilitate remote access to his medical records and connect with his health provider via a telehealth portal. This allows the exchange of information (e.g., pictures/images of bruises), dissemination of advice/recommendations as well as the review of patient's clinical outcomes remotely (e.g., assessment of physical function, and joint damage following a bleeding episode).

\subsection{The HemPHL App Design}

Table 1 shows a thematic assessment of the user requirements in the above scenarios. Each user requirement is mapped to one or more features (Table 2) to enable patients or caregivers perform the desired actions. Table 2 shows the main interface features of the proposed PHL through which patients can set their preferences to tailor the content in the targeted recommendations for self-management (e.g., medication adherence, prescriptions, resource suggestions), frame (e.g., educational, motivational, goal-based), frequency (e.g., daily, weekly). The app can personalize these recommendations further by taking into account ODL data through device readings (e.g., a Fitbit) for physical activity/functioning as well patient's location e.g. use of zip code area for specifying Hemophilia Treatment Centers, clinics, physicians, or other resources. 
Table 1. Requirement analysis for HemPHL features on specified case scenarios

\begin{tabular}{|l|l|}
\hline Requirements & \multicolumn{1}{c|}{ Descriptions } \\
\hline Scenario 1 & $\begin{array}{l}\text { The ability to (a) educate, find, use information from trusted sources of knowledge, (b) } \\
\text { provide telehealth, (c) record and monitor bleeding events (d) track medication adherence }\end{array}$ \\
\hline Scenario 2 & $\begin{array}{l}\text { The mechanism to (a) add/subscribe to trusted domains e.g. Facebook, (b) protect personal } \\
\text { data/information, (c) add the web of trust e.g. caregiver, friends (d) add trusted devices, } \\
\text { applications }\end{array}$ \\
\hline Scenario 3 & $\begin{array}{l}\text { The mechanism to (a) provide telehealth (b) add trusted contacts e.g. physical therapist } \\
\text { (b) support REST API }\end{array}$ \\
\hline
\end{tabular}

REST (Representational State Transfer); API (Application Programming Interface)

Overall, this platform support patients' management of bleeding episodes, and adherence to self-administered prophylactic factor infusions and other medications.

Table 2. Main features of the HemPHL App for Hemophilia Self-Management

\begin{tabular}{|l|l|}
\hline Features & \multicolumn{1}{|c|}{ Components } \\
\hline Customization & Manage, utilize information based on personal preferences/choices \\
\hline $\begin{array}{l}\text { Health information \& } \\
\text { personal care }\end{array}$ & $\begin{array}{l}\text { Nutrition/diet, health and fitness, medication adherence, medical complications } \\
\text { \& emergencies, genetics, podcasts (educational, motivational, spiritual). }\end{array}$ \\
\hline $\begin{array}{l}\text { Electronic medical } \\
\text { records }\end{array}$ & $\begin{array}{l}\text { Laboratory blood tests, radiological imaging e.g. X-rays, medical and family } \\
\text { histories, allergies, treatment therapies, etc. }\end{array}$ \\
\hline $\begin{array}{l}\text { Recall, Reminders, } \\
\text { Alerts, Notifications }\end{array}$ & $\begin{array}{l}\text { Medication diaries and logs, vaccinations updates e.g. hepatitis B, clinical } \\
\text { appointments/check-ups, blood testing/screenings e.g. bloodborne infections }\end{array}$ \\
\hline Self-monitoring & Bleeding episodes, pain, swelling, bruises, nosebleeds, physical functioning etc. \\
\hline $\begin{array}{l}\text { Observations of daily } \\
\text { living (ODL) }\end{array}$ & Physical activity, heart rate, blood pressure, pulse \\
\hline $\begin{array}{l}\text { Patient-provider } \\
\text { communications }\end{array}$ & Telehealth, video conferencing \\
\hline Treatment/resources & Directory for nearest hemophilia treatment centers, clinics \\
\hline Mental health & Online counseling therapy, support group, chats \\
\hline Support groups & Chats etc. \\
\hline Weblinks & $\begin{array}{l}\text { CDC (e.g. Community counts), National Hemophilia Foundation's National } \\
\text { Prevention Program, etc., peer-reviewed scientific papers }\end{array}$ \\
\hline $\begin{array}{l}\text { Drug safety reporting } \\
\text { system }\end{array}$ & Reports adverse drug events/reactions \\
\hline Surveillance system & Registry for hemophilia surveillance \\
\hline $\begin{array}{l}\text { Others } \\
\text { Language and subscription preferences, music \& games, supports voice- } \\
\text { activated technologies, enables data breach notification }\end{array}$ \\
\hline
\end{tabular}

\subsection{Evaluation}

For a formative evaluation, a panel of subject matter experts will assess the HemPHL app for ease of usability, clarity, workflow, clinical content, requirement gathering, omission, etc., by utilizing (i) semi-structured interviews to obtain qualitative data and (ii) completion of a System Usability Scale (SUS). Thereafter, the effectiveness of the app in promoting self-management and home-based therapy among targeted patients is evaluated through assessment and comparison of study outcome measures at baseline, after 6- and 12- months. The outcome measures of interest for this study include patient's (i) knowledge on hemophilia and treatment strategies (ii) clinical outcomes e.g., frequency of emergency room visits, bleeding episodes (iii) health-related quality of life e.g., physical functioning following joint bleed, mental health status, adherence to prophylactic infusions, etc. and (iv) satisfaction with PHL platform. 


\section{Conclusion}

Long-term management of hemophilia could be complex requiring an all-inclusive, integrated, and multidisciplinary approach. HemPHL can provide a digital health monitoring system and real-time recommendations which facilitate improved efficiency in patient's self-management through informed health decision-making. Data such as EMRs, ODL readings, and SDoH are transformed into knowledge with semantics which allows users to generate personalized insight. The adoption of digital health technologies could eliminate the limitations in timely, recurring access to medical care and information, reduce associated travel costs and hospital visits as well as empower patients to control their health. The process of enabling patients to remotely monitor their health progress, take greater responsibility for their care, and have access to personal health information could optimize patient's satisfaction, treatment regimen, clinical outcomes, and overall quality of life.

\section{References}

[1] Rangarajan S, Walsh L, Lester W, Perry D, Madan B, Laffan M, Yu H, Vettermann C, Pierce GF, Wong WY, Pasi KJ. AAV5-Factor VIII Gene Transfer in Severe Hemophilia A. N Engl J Med. 2017 Dec 28;377(26):2519-2530.

[2] Curtis R, Baker J, Riske B, Ullman M, Niu X, Norton K, Lou M, and Nichol MB. Young adults with hemophilia in the U.S.: demographics, comorbidities, and health status. Am. J. Hematol. 2015 Nov; 90 : s11-16.

[3] Chen SL. Economic costs of hemophilia and the impact of prophylactic treatment on patient management. Am J Manag Care. 2016 Apr;22(5 Suppl): s126-33.

[4] Chen CX, Baker JR, Nichol MB. Economic burden of illness among persons with hemophilia B from HUGS Vb: examining the association of severity and treatment regimens with costs and annual bleed rates. Value in Health. 2017 Sep 1;20(8):1074-82.

[5] McGowan PT. Self-management education and support in chronic disease management. Prim Care. 2012 Jun;39(2):307-25.

[6] Mazepa MA, Monahan PE, Baker JR, Riske BK, Soucie JM; US Hemophilia Treatment Center Network. Men with severe hemophilia in the United States: birth cohort analysis of a large national database. Blood. 2016 Jun 16;127(24):3073-81.

[7] Qian W, Lam TT, Lam HH, Li CK, Cheung YT. Telehealth interventions for improving self-management in patients with hemophilia: Scoping review of clinical studies. Journal of medical Internet research. 2019;21(7):e12340.

[8] Ammar N, Bailey JE, Davis RL, Shaban-Nejad A. The Personal Health Library: A Single Point of Secure Access to Patient Digital Health Information. Stud Health Technol Inform. 2020 Jun 16; 270:448-452. doi:10.3233/SHTI200200.

[9] Ammar N, Bailey J, Davis RL, and Shaban-Nejad A. Implementation of a Personal Health Library (PHL) To Support Self-Management of Chronic Diseases. in Explainable AI in Healthcare and Medicine. Studies in Computational Intelligence, Springer/ Nature, Switzerland AG, 2020.

[10] Ammar N, Bailey JE, Davis RL, Shaban-Nejad A. The Personal Health Library for Enabling an mHealth Recommender System for Self-Management of Diabetes Among Underserved Populations with Multiple Chronic Conditions: Use Case for Knowledge Graphs and Linked Data. JMIR Form Res 2021.

[11] Ammar N, Shaban-Nejad A. Explainable Artificial Intelligence Recommendation System by Leveraging the Semantics of Adverse Childhood Experiences: Proof-of-Concept Prototype Development. JMIR Med Inform. 2020 Nov 4;8(11):e18752. doi: 10.2196/18752. 